\title{
Alterations of intestinal lipoprotein metabolism in diabetes mellitus and metabolic syndrome
}

\author{
Marcello Arca* \\ Department of Internal Medicine and Medical Specialties, UOS Atherosclerosis Center, La Sapienza University of Rome, Rome, Italy
}

\begin{abstract}
Diabetes and metabolic syndrome are associated with abnormal postprandial lipoprotein metabolism, with a significant delay in the clearance of many lipid parameters, including triglycerides and chylomicrons. Abnormal concentrations of plasma lipids can result from changes in the production, conversion, or catabolism of lipoprotein particles. Whereas the liver is involved in controlling serum lipid levels through synthesis of liver derived triglyceride-rich lipoproteins and low-density lipoprotein metabolism, the intestine also has a major role in lipoprotein production. Postprandial lipemia results from increases in apoB-48 availability, lipogenesis, and the synthesis and absorption of cholesterol in the enterocytes. Increased intestinal lipoprotein production prolongs postprandial lipemia in patients with diabetes and MetS, and may contribute directly to atherogenesis in these patients.
\end{abstract}

(c) 2015 Elsevier Ireland Ltd. All rights reserved.

Keywords: Diabetes mellitus; Metabolic syndrome; Intestine; Cholesterol metabolism; Postprandial lipemia

\section{Abnormalities of lipoprotein metabolism in diabetes mellitus and metabolic syndrome}

Dyslipidemia associated with diabetes mellitus and metabolic syndrome (MetS) is characterized by a cluster of metabolically interrelated lipoprotein abnormalities. Due to the elevated atherogenic potential of these abnormalities they are usually comprehensively designated as atherogenic dyslipidemia (AD). The phenotypic hallmarks of $\mathrm{AD}$ are increased plasma triglycerides (TG), reduced high-density lipoprotein cholesterol (HDL-C) and increased numbers of small, dense low-density lipoprotein (sdLDL) particles.

Abnormal concentrations of plasma lipids can result from changes in the production, conversion, or catabolism of lipoprotein particles. Therefore, several studies have investigated the kinetic parameters of apoB-containing lipoproteins [low-density lipoprotein (LDL) and very-low-

\footnotetext{
* Department of Internal Medicine and Medical Specialties, UOS Atherosclerosis Center, Sapienza University of Rome, Policlinico Umberto I, Viale del Policlinico 155 - 00161 Rome, Italy. Tel.: +39 064451354; fax: +69 064463534 .

E-mail address: marcello.arca@uniroma1.it.
}

density lipoprotein (VLDL)] to identify the mechanisms responsible for $\mathrm{AD}$. These studies have consistently reported that the major metabolic defects in AD are increased VLDL production, and reduced catabolism of LDL and its precursor, intermediate-density lipoprotein (IDL).

Overproduction of VLDL is particularly evident in patients with diabetes, where this abnormality is related to the plasma glucose level and indices of tissue insulin sensitivity such as the HOMA index. In addition, intraabdominal and liver fat are also significant predictors of liver production of large TG-rich VLDL [1].

Assessment of HDL metabolism in subjects with diabetes using a stable isotope method revealed a higher than normal mean fractional catabolic rate (FCR) of apoAIHDL, strongly suggesting that HDL is catabolized more rapidly in these patients. Therefore, the AD phenotype can result from: 1) increased production of VLDL, 2) a reduced catabolic rate of apoB-containing lipoproteins (IDL and LDL), and 3) an increased catabolic rate of HDL.

The liver plays a fundamental role in cholesterol homeostasis, because it processes cholesterol taken up from plasma lipoproteins and chylomicrons, and uses cholesterol to form bile acids that are subsequently secreted into the bile. As the major site of LDL catabolism, the liver also 
has a part in determining plasma LDL levels. As described elsewhere in this supplement [2], the liver is not the only organ with an important role in this process: also the intestine plays an essential role. In diabetes mellitus or MetS, chylomicrons produced in the intestine can accumulate in the circulation thereby influencing overall lipid and lipoprotein turnover. There are indications that chylomicrons and cholesterol metabolism in the intestine are involved in regulating plasma LDL concentrations [3].

\section{Abnormalities in intestinal lipids and lipoprotein metabolism in diabetes and metabolic syndrome}

Patients with diabetes have abnormal post absorptive lipoprotein metabolism, with a significant delay in the postprandial clearance of many lipid parameters, including triglycerides and chylomicrons, in spite of normal fasting levels [4]. Similar results have been observed in people with MetS [5]. This is observed even if subjects are categorized according to insulin resistance.

Chylomicrons are lipoprotein particles secreted by the intestine during the postprandial period. Their assembly is a complex, multistep process (Fig. 1), in which apoB48 synthesis is one of the limiting factors [6]. Duez et al. hypothesized that abnormalities in the synthesis of intestinally-derived apolipoproteins drive the prolonged postprandial phase in insulin-resistant subjects, and examined the relationship between insulin resistance and the rate of production of intestinal lipoproteins [7]. They found that ApoB-48 was produced at a significantly higher rate in hyperinsulinemic, insulin-resistant subjects and that this correlated with fasting plasma insulin concentrations. Consistent with this observation, others have reported that insulin administration decreases levels of circulating apoB48-containing lipoproteins [8].
More recent evidence indicates that the adipokine resistin is also involved in regulating chylomicron assembly. Plasma resistin concentrations are elevated in MetS and associated with insulin resistance [9]. In cultured hepatocytes, resistin stimulates the overproduction of VLDL apo-B by increasing the activity of the microsomal transfer protein (MTP), which plays a pivotal role in coupling triglycerides with apoB-48 in chylomicrons (Fig. 1), and by decreasing insulin signaling [10].

Chylomicron assembly is also influenced by the availability of cholesterol in enterocytes. Regulation of chylomicron cholesterol is depicted in Fig. 2. Cholesterol is absorbed through a process facilitated by the NiemannPick C1-Like 1 (NPC1L1) protein, which plays the major quantitative role in intestinal cholesterol absorption. In addition, ABCG5 and ABCG8 proteins regulate enterocyte cholesterol content by promoting its re-excretion into the intestinal lumen throughout the intestinal villi.

NPC1L1 mRNA expression is elevated in patients with type 2 diabetes [11], suggesting that cholesterol absorption is increased. Moreover, these patients also have increased expression of MTP, associated with reduced expression of the cholesterol efflux transporters ABCG5 and ABCG8 (Fig. 3). Taken together, this pattern strongly support the notion that diabetic patients have elevated amounts of enterocyte cholesterol available for chylomicron assembly.

Based on these observations, one could hypothesize that the modulation of intestinal cholesterol metabolism might be a useful strategy for controlling the exaggerated postprandial lipemia in insulin-resistant conditions (diabetes mellitus and MetS). Ezetimibe is a compound that specifically reduces intestinal cholesterol absorption by inhibiting the activity of NPC1L1 [12]. Administration of ezetimibe to men with moderate primary hypercholesterolemia increases the fractional catabolic rate of apoB-100-containing

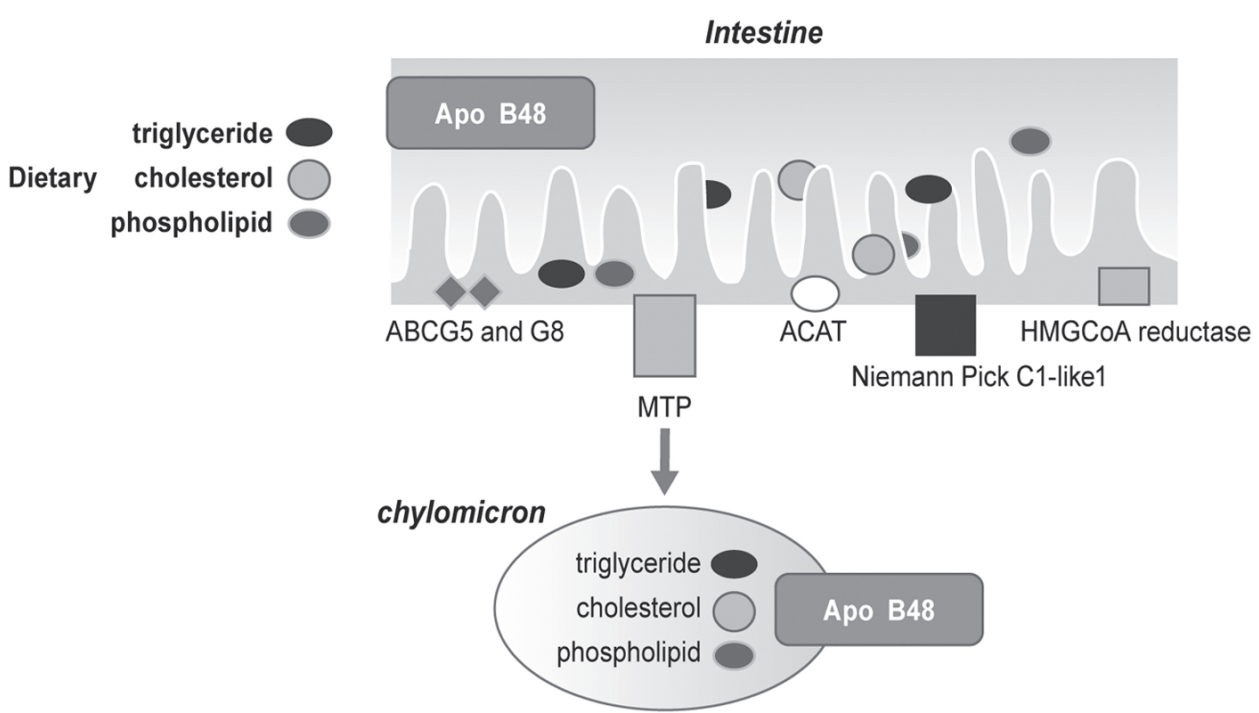

Fig. 1. Regulation of chylomicron synthesis. ApoB-48, apoproptein B-48; ABCG, ATP-binding cassette sub-family G member; ACAT, AcylCoA:cholesterol acyltransferase; HMGCoA, hydroxy-3-methyl-glutaryl-CoA. (Reproduced with permission from Diabetes Care 2008; 31(Suppl. 2): S241-8.) 


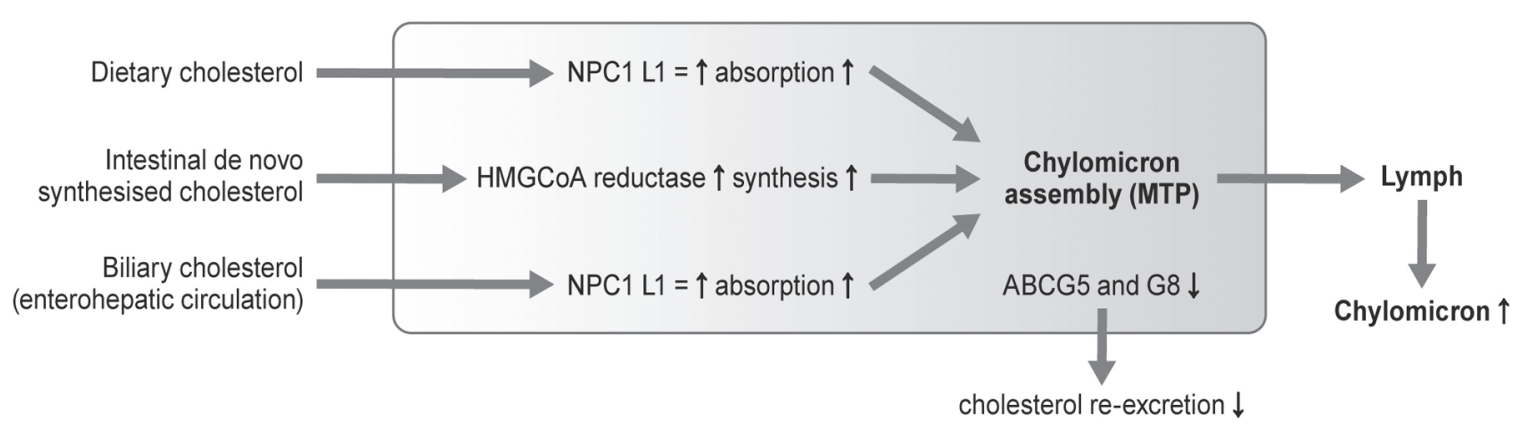

Fig. 2. Chylomicron cholesterol derives from dietary, biliary and intestinal de novo synthesized cholesterol. NPC1-L1 regulates its absorption and ABCG5 and ABCG8 regulate its excretion. MTP assembles the apoB-48 protein, cholesterol and other lipids to form the chylomicron, which is then secreted into the lymph. (Reproduced with permission from Diabetes Care 2008 Feb;31 Suppl 2:S241-8 [6].)

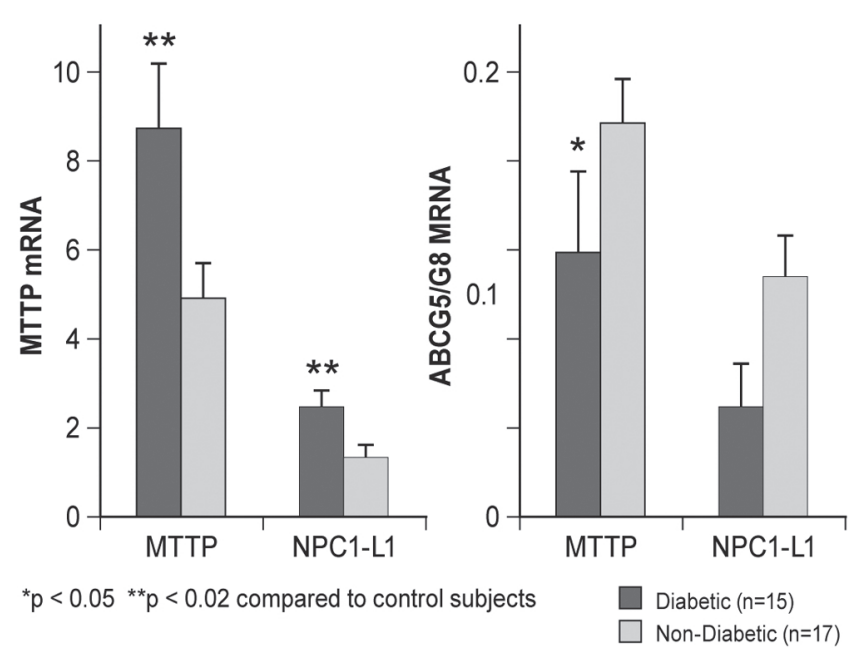

Fig. 3. Comparison of mRNA expression of intestinal proteins regulating cholesterol absorption and chylomicron composition in diabetic $(n=15)$ (black bars) and non-diabetic control patients $(n=17)$ (white bars) not receiving statin treatment. a) microsomal triglyceride transfer protein (MTP) and Niemann-Pick C1-like 1 (NPC1-L1); b) ATP-binding cassette, transporters G5 and G8 (ABCG5/G8). Mean \pm SE, ${ }^{*} p<0.05$, $* * p<0.02$ compared to control subjects. (Reproduced with permission from Diabetologia 2006 May;49(5):1008-16 [11].)

lipoproteins, with an associated decrease in this lipoprotein fraction [13], which is considered the major mechanism of action of this drug. However, several studies have demonstrated that ezetimibe has a beneficial effect also on postprandial triglyceride-rich lipoproteins in patients with type 2 diabetes. For example, postprandial chylomicron apoB-48 concentrations were about $50 \%$ lower in subjects with type 2 diabetes and hypercholesterolemia receiving simvastatin + ezetimibe, compared with simvastatin + placebo (Fig. 4) [14]. This decrease suggests that ezetimibe reduces the number of intestinal particles, confirming the results from the kinetic study in men with mixed hyperlipidemia cited above [13].

Chylomicron cholesterol content was also significantly lower after ezetimibe compared to placebo, even in the fasting state, and this difference remained statistically significant during the entire postprandial phase. Postprandial levels of chylomicron triglycerides and the cholesterol/ triglyceride ratio were also significantly lower with ezetimibe, compared to placebo, as were the levels of apoB.

\section{Contribution of insulin resistance-related perturbation of intestinal lipid metabolism to cardiovascular risk in patients with diabetes or metabolic syndrome}

During the postprandial phase, triglyceride-rich lipoproteins (chylomicrons and VLDL) are converted into partially hydrolyzed lipoproteins known as remnant-like particles (RLPs) which represent transient lipoprotein particles relatively enriched in apoB and cholesterol. Therefore, exaggerated postprandial lipemia may favor the production of elevated amounts of RLPs. Several epidemiological studies have indicated that RPLs significantly contribute to atherogenesis. The Honolulu Heart Study [15], a 17-year prospective study that followed a cohort of 1156 JapaneseAmerican men aged 60 to 82, documented 164 incident cases of CHD, and found that elevated RLPs levels were associated with a 2-fold increase in CHD risk after adjustment for potential confounding variables.

Finally, there are direct demonstrations that RLPs can be retained in arterial vessels, and that this phenomenon is modulated by insulin sensitivity. Mangat et al. investigated arterial retention of intestinal-derived remnants using labeled RLPs in an ex vivo system in the JCR:LA-cp rat model of insulin resistance, in order to identify potential mechanisms of exacerbated cholesterol deposition in insulin resistance [16]. They found significantly more retention in hyperinsulinemic animals, suggesting that the insulin resistant state adversely affect the interaction of lipoprotein metabolism with the arterial wall. However, arterial RLP retention was significantly reduced when hyperinsulinemic animals were treated with ezetimibe or ezetimibe+simvastatin. These observation indicate that the arterial retention of cholesterol-rich remnants may explain part of the association between insulin resistant states (diabetes mellitus and MetS) and atherosclerosis, and suggests that ezetimibe alone or in combination with a statin could reduce the risk of atherosclerotic vascular damage under these conditions. 


\section{Chylomicrons}
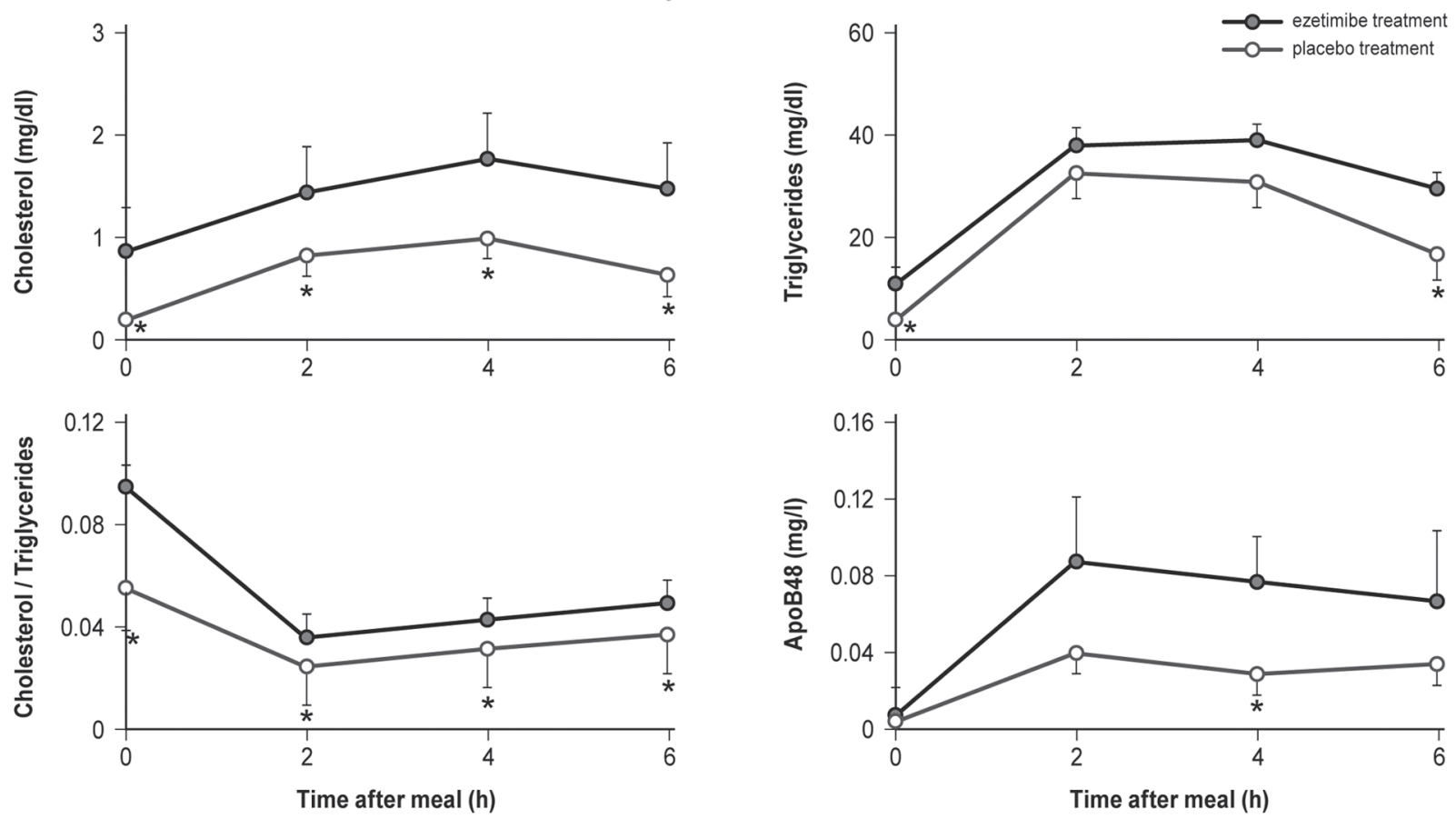

Fig. 4. Postprandial cholesterol, triglycerides, cholesterol/triglyceride ratio, and apoB-48 concentrations in chylomicrons with ezetimibe and placebo treatment in patients with type 2 diabetes. ${ }^{*} p<0.05$ by paired samples $t$-test. (Reproduced with permission from Atherosclerosis. 2011 Jul;217(1):142-8 [14].)

\section{Conclusions}

Atherogenic dyslipidemia is very common in insulin resistant conditions such as diabetes and MetS. In addition to increased hepatic synthesis of liver derived triglyceriderich lipoproteins (VLDL), these conditions are associated with increased intestinal lipoprotein production. This results from increases in apoB-48 availability, lipogenesis, and the synthesis and absorption of cholesterol in the enterocytes. Increased intestinal lipoprotein production prolongs postprandial lipemia in patients with diabetes and MetS, and may contribute directly to atherogenesis in these patients.

\section{Disclosure}

Dr. Arca received lecture honoraria, consultancy fees and research funding from Merck Sharp \& Dohme, Pfizer, Asta Zeneca, Kowa, Sanofi, Regeneron, Roche, Genzyme, Abbott, Boheringer, Aegerion, Mediolanum, SigmaTau, Menarini, ISIS, Amgen.

This work was funded by an unrestricted grant by MSD Italia Srl. The sponsor had no role in reviewing the literature, drafting or reviewing the paper, or in the decision to submit the manuscript for publication. All views expressed are solely those of the author. The author would like to thank Editamed Srl for editorial assistance in the preparation of the manuscript.

\section{References}

[1] Adiels M, Borén J, Caslake MJ, Stewart P, Soro A, Westerbacka J, Wennberg B, Olofsson SO, Packard C, Taskinen MR. Overproduction of VLDL1 driven by hyperglycemia is a dominant feature of diabetic dyslipidemia. Arterioscler Thromb Vasc Biol. 2005 Aug;25(8):1697-703.

[2] Moschetta A. Nuclear receptors and cholesterol metabolism in the intestine. Atheroscler Suppl 2015;16:9-11 (this issue).

[3] Kesäniemi YA, Miettinen TA. Cholesterol absorption efficiency regulates plasma cholesterol level in the Finnish population. Eur J Clin Invest 1987 Oct;17(5):391-5.

[4] Rivellese AA, De Natale C, Di Marino L, Patti L, Iovine C, Coppola S, Del Prato S, Riccardi G, Annuzzi G. Exogenous and endogenous postprandial lipid abnormalities in type 2 diabetic patients with optimal blood glucose control and optimal fasting triglyceride levels. J Clin Endocrinol Metab 2004 May;89(5):2153-9.

[5] Kolovou GD, Anagnostopoulou KK, Pavlidis AN, Salpea KD, Iraklianou SA, Tsarpalis K, Damaskos DS, Manolis A, Cokkinos DV. Postprandial lipemia in men with metabolic syndrome, hypertensives and healthy subjects. Lipids Health Dis 2005 Sep 30;4:21.

[6] Tomkin GH. Targets for intervention in dyslipidemia in diabetes. Diabetes Care 2008 Feb;31 Suppl 2:S241-8.

[7] Duez H, Lamarche B, Uffelman KD, Valero R, Cohn JS, Lewis GF. Hyperinsulinemia is associated with increased production rate of intestinal apolipoprotein B-48-containing lipoproteins in humans. Arterioscler Thromb Vasc Biol 2006 Jun;26(6):1357-63.

[8] Federico LM, Naples M, Taylor D, Adeli K. Intestinal insulin resistance and aberrant production of apolipoprotein B48 lipoproteins in an animal model of insulin resistance and metabolic dyslipidemia: evidence for activation of protein tyrosine phosphatase-1B, extracellular signal-related kinase, and sterol regulatory elementbinding protein-1c in the fructose-fed hamster intestine. Diabetes 2006 May;55(5):1316-26. 
[9] Hivert MF, Sullivan LM, Fox CS, Nathan DM, D'Agostino RB Sr, Wilson PW, Meigs JB. Associations of adiponectin, resistin, and tumor necrosis factor-alpha with insulin resistance. J Clin Endocrinol Metab 2008 Aug;93(8):3165-72.

[10] Costandi J, Melone M, Zhao A, Rashid S. Human resistin stimulates hepatic overproduction of atherogenic ApoB-containing lipoprotein particles by enhancing $\mathrm{ApoB}$ stability and impairing intracellular insulin signaling. Circ Res 2011 Mar 18;108(6):727-42.

[11] Lally S, Tan CY, Owens D, Tomkin GH. Messenger RNA levels of genes involved in dysregulation of postprandial lipoproteins in type 2 diabetes: the role of Niemann-Pick C1-like 1, ATP-binding cassette, transporters G5 and G8, and of microsomal triglyceride transfer protein. Diabetologia 2006 May;49(5):1008-16.

[12] Altmann SW, Davis HR Jr, Zhu LJ, Yao X, Hoos LM, Tetzloff G, Iyer SP, Maguire M, Golovko A, Zeng M, Wang L, Murgolo $\mathrm{N}$, Graziano MP. Niemann-Pick C1 Like 1 protein is critical for intestinal cholesterol absorption. Science 2004 Feb 20;303(5661): $1201-4$.

[13] Tremblay AJ, Lamarche B, Cohn JS, Hogue JC, Couture P. Effect of ezetimibe on the in vivo kinetics of apoB-48 and apoB-100 in men with primary hypercholesterolemia. Arterioscler Thromb Vasc Biol 2006 May;26(5):1101-6.

[14] Bozzetto L, Annuzzi G, Corte GD, Patti L, Cipriano P, Mangione A, Riccardi G, Rivellese AA. Ezetimibe beneficially influences fasting and postprandial triglyceride-rich lipoproteins in type 2 diabetes. Atherosclerosis $2011 \mathrm{Jul} ; 217(1): 142-8$.

[15] Imke C, Rodriguez BL, Grove JS, McNamara JR, Waslien C, Katz AR, Willcox B, Yano K, Curb JD. Are remnant-like particles independent predictors of coronary heart disease incidence? The Honolulu Heart study. Arterioscler Thromb Vasc Biol 2005 Aug;25(8):1718-22.

[16] Mangat R, Warnakula S, Borthwick F, Hassanali Z, Uwiera RR, Russell JC, Cheeseman CI, Vine DF, Proctor SD. Arterial retention of remnant lipoproteins ex vivo is increased in insulin resistance because of increased arterial biglycan and production of cholesterol-rich atherogenic particles that can be improved by ezetimibe in the JCR:LA-cp rat. J Am Heart Assoc 2012 Oct;1(5): e003434. 